\title{
Awareness of Complications of Obesity in Young Individuals
}

\author{
Anto Gnana Delasalle $\mathrm{M}^{1}$, $(\mathrm{Col})$ Arunachalam ${ }^{2}$, Sharol Asma Menezes ${ }^{3}$ \\ ${ }^{I}$ PG Resident, Department of General Medicine, Father Muller Medical College, Mangalore \\ ${ }^{2}$ Professor, Department of General Medicine, Father Muller Medical College, Mangalore \\ ${ }^{3}$ Senior Resident, Department of General Medicine, Father Muller Medical College, Mangalore
}

\begin{abstract}
Obesity is a leading cause of morbidity worldwide. As per WHO data in 2008, 1.4 billion persons aged 20 years and above were obese. Type 2 diabetes mellitus, hypertension, dyslipidemia, ischemic heart disease, stroke, gall bladder stones, osteoarthritis and nonalcoholic fatty liver disease are some of the common complications of obesity. Awareness of these complications is very essential among young individuals. The study included a 100 individuals attending out-patient services of Father Muller Medical College Hospital by convenience sampling. Most participants were aware about complications of obesity such as joint pains, risk of developing diabetes, hypertension, hypercholesterolemia, coronary artery disease, asthma, obstructive sleep apnea and depression. There was no significant body image misperception among the participants in this study. Many participants in our study felt that obesity has a genetic factor because of which obesity cannot be prevented. Community wide efforts to increase awareness and promote environments that encourage physical activity and healthy nutrition are needed. These measures could probably reduce this misperception.
\end{abstract}

Keywords: Obesity, Awareness of complications, Diabetes Mellitus, Metabolic Syndrome, Dyslipidemia

\section{Introduction And Need For The Study}

Obesity is a leading cause of morbidity worldwide. As per WHO data in 2008, 1.4 billion persons aged 20 years and above were obese. ${ }^{(1)}$ Obesity in India and other developing countries is on an increasing trend, as a result of rapid urbanization and changing lifestyles. ${ }^{(2)}$ There are various factors that play a role in causing obesity. Obesity itself can lead to multiple complications. Type 2 diabetes mellitus, hypertension, dyslipidemia, ischemic heart disease, stroke, gall bladder stones, osteoarthritis and nonalcoholic fatty liver disease are some of the common complications of obesity. ${ }^{(3)}$ Awareness of these complications is very essential among young individuals. Very few studies have been done on awareness of obesity and its complications in young individuals in Mangalore. Hence this study attempts to study the awareness of complications of obesity in young individuals.

\section{Review of Literature}

Obesity is known to be associated with multiple diseases affecting multiple organ systems. The major diseases associated with obesity are diabetes mellitus, hypertension, dyslipidemias, atherosclerotic disease and cancers. These complications are fairly well known among the general public. Other complications such as cholelithiasis, impairment of pulmonary function, sleep disorders, endocrine abnormalities, joint disorders, dermatologic disorders and immunologic impairment are not so well known among people not associated with health care. ${ }^{(3)}$ Complications of obesity in childhood include psychosocial problems, learning impairment, abnormal glucose tolerance, hypertension and significant risk of obesity during adulthood and beyond. ${ }^{(4)}$

A study done by Gregory $\mathrm{CO}$ et al found that men were more likely than women to disagree their body weight was a health risk. Disagreement with risk was associated with good health status and race/ethnicity among both sexes and lower education and income among women. ${ }^{(6)} \mathrm{A}$ study done in Europe showed that despite a tendency of lower interest in healthy eating among obese consumers, interest in healthy eating was not significantly associated with the likelihood of being obese or overweight. ${ }^{(7)} \mathrm{A}$ focus group study done in the state of Georgia (USA) found that more overweight youth attributed being overweight to external causes, such as slow metabolism or genetics, rather than alterable lifestyle behaviors, such as diet and physical activity. They also did not tend to see sustained lifestyle change as desirable or achievable.$^{(8)}$ Bruce SG et al from the University of Manitoba concluded that the prevalence of obesity and its complications in Canada was higher among women than men. Most common complications of obesity were dyslipidemia, hypertension and diabetes. This study also showed that the complications of obesity was higher among younger individuals between the age group of 18 to 29 years. ${ }^{(9)}$

\subsection{Source of data:}

\section{Materials And Methods}

The data was collected from young individuals attending outpatient and inpatient services in Father Muller Medical College Hospital for a period of 3 months from January 2016 to March 2016. 


\subsection{Method of collection of data: Study Design}

This is a prospective study on awareness of complications of obesity in young individuals attending the outpatient services in Father Muller Medical College Hospital. The study included 100 individuals by convenience sampling A detailed questionnaire was given to all subjects to collect information regarding demography, socioeconomic status, behavioural and health status of the individual. Questionnaire evaluated the knowledge regarding the complications of obesity.

\section{Inclusion Criteria:}

Subjects between the age 18 and 30 years.

\section{Exclusion Criteria}

Individuals directly related with providing health care like doctors, nurses, medical students.

Data analysis:

Data was analyzed by frequency, proportions and Chi-square tests using SPSS V20.0

\section{Results}

The study was done on 100 volunteers. Equal number of males and females opted to be a part of this study. $66 \%$ of respondents were in the age group 24 to $26,27 \%$ between 20 to 23 years of age and the rest 25 years or older. 85 respondents reported to be from middle class, 7 from lower class and 8 from higher class. $7 \%$ of respondents were underweight ( 3 males and 4 females), 54\% were within the normal BMI range (28 males and 26 females), $18 \%$ were overweight ( 8 males and 10 females), $17 \%$ were obese (9males and 8 females), 2 females were morbidly obese and 2 males were super obese.

Participants were interviewed about their food habits and lifestyle. $71 \%$ (36 male, 35 female) of respondents reported to consume 1-3 servings of fruits per day, 15\% (7 male, 8 female) reported to consume 4 or more servings of fruits per day, whereas $13 \%$ ( 7 males, 6 females) did not consume fruits on a regular basis. $26 \%$ of respondents reported to consume more than 4 servings of vegetables per day, only $7 \%$ (all 7 were males) reported that they did not consume vegetables on a day to day basis (p-0.007). 56\% (27 males, 29 females) reported that they do not consume fast food on a regular basis per week. 32\%(14 males, 18 females) reported to consume fast food atleast once per week whereas only 12\%(9 males and 3 females) reported to have a higher rate of fast food consumption. 62\% (33 males and 29 females) reported to not have a habit of soft drinks consumption. 29\% (12 males and 17 females) reported that they consume 1-4 soft drinks per week. The rest i.e $9 \%$ reported to have a higher rate of soft drinks consumption. 35\% (18 males and 17 females) reportedly exercised for atleast 20 min on 1-2 days per week. $27 \%$ (19 males and 8 females) exercised for 20 min on 3-4 days per week and 25\% (7 males and 18 females) reported to never exercise over the entire week. This difference was statistically significant (p-0.049). 41\%(22 females and 16 males) reported to watch TV or use computer for 1-2 hrs everyday.

The volunteers were then asked about their awareness about the factors resulting in obesity. $76 \%$ felt that obesity has a genetic cause and that there is nothing much one can do to prevent obesity. A higher percentage i.e $81 \%$ of participants felt that what one eats also could make a big difference to the odds of one getting a disease. Factors influencing one's decision when they shop for food items or eat outside were then assessed by a set of questions. Food safety $(94 \%)$ and nutritive value $(96 \%)$ seemed to be a priority to most participants. Price of food products was also a priority to most participants (69\%).

They were then asked about the factors which they thought were important for their present and future health. The volunteers felt that exercise ( $88 \%$ of participants), What one eats ( $87 \%$ of participants), How much one eats (83\% of participants) and how much one weighs ( $80 \%$ of participants) were all important. Participants were asked about the awareness of obesity and its complications. $89 \%$ felt that obese people have a risk of developing joint pains. $74 \%$ felt that obese people have a higher risk of developing life threatening diabetes. $72 \%$ felt that obese people have a higher risk of developing respiratory problems like asthma whereas only $14 \%$ felt that obese persons have a higher of developing skin infections. $68 \%$ participants were aware that obesity could cause problems with sleeping due to Obstructive sleep apnea. $85 \%$ believed that obese children are bullied in school which could force them into depression. Only 57\% felt that obese persons are prone for psychological stress which could affect their learning. $40 \%$ of participants felt that obese persons are socially isolated. $86 \%$ participants felt that obesity is associated with higher blood cholesterol levels. $82 \%$ felt that obese persons have a higher risk of developing fatally high blood pressure levels, $65 \%$ felt that obese persons develop angina symptoms more frequently than non-obese persons, a lesser proportion i.e $61 \%$ felt that the most common cause of death in obese persons is due to blockage of blood vessels in the heart (Coronary artery disease). $72 \%$ felt that obese persons have a shorter lifespan (by atleast 7 years) when compare to non-obese individuals. $75 \%$ of participants replied in the affirmative when asked whether obesity was caused due to food habits, sedentary lifestyle and lack of knowledge about obesity. $72 \%$ felt that obesity could be prevented if appropriate changes 
were made to one's eating habits and lifestyle. There were no significant differences between the responses of males and females to the above questions.

\section{Discussion}

In our study most participants were aware about complications of obesity such as joint pains, risk of developing diabetes, hypertension, hypercholesterolemia, coronary artery disease, asthma, obstructive sleep apnea and depression. Also most of the study participants believed that obese individuals do have a shorter life span compared to non-obese individuals. A fewer percentage of participants felt that obese individuals have a higher risk of skin infections and are socially isolated. The above findings show that most adults are aware of the problems due to obesity.

In contrast to previous studies which showed that obese individuals were most likely to underestimate their weight, our study showed that obese individuals seemed to accept that they were indeed overweight and needed to take steps for weight reduction. We also found that individuals with their BMI in the high normal range (22.5-22.9) felt that they were overweight or obese. This is probably related to the fact that they were aware of the complications of obesity and hence felt that weight reduction was needed to prevent those complications. There was no significant body image misperception among the participants in this study.

Many participants in our study felt that obesity has a genetic factor because of which obesity cannot be prevented. This is a dangerous perception. The belief in an external (and potentially non-modifiable) locus of control is particularly concerning because this belief has previously been correlated with continued weight gain into adulthood. ${ }^{(10)}$ Hence spreading awareness about obesity and preventive measures should be started in childhood. Schools should provide daily physical education and frequent periods of unstructured play in young children. Clinical treatment should be both encouraged and financially supported in children who are already overweight. Community wide efforts to increase awareness and promote environments that encourage physical activity and healthy nutrition are needed. ${ }^{(11)}$ These measures could probably reduce this misperception. Unlike previous studies which showed that obese individuals were more likely to attribute obesity to non-modifiable risk factors, our study showed that obesity was equally attributed to both modifiable and non-modifiable factors. This showed a better awareness among participants about the etiology of obesity.

\section{Limitations}

Our study was done among apparently healthy adults visiting the out patient and in patient services of our hospital. Since this study was a hospital based study, there is high likelihood that responses had some component of social desirability bias. A community based study would have been more appropriate. Sample size was limited due to want of resources. Number of obese participants were also limited. Participants interviewed were not followed up to look for lifestyle modifications (cross-sectional study).

\section{Conclusion}

Most participants were aware about obesity and most of its complications. Participants with a high normal BMI were more frequently found to over-estimate their weight. This probably is a healthy behavior as this would lead them to modify their lifestyle, leading to further weight reduction. Though many of the participants were of the view that obesity is a modifiable risk factor for many lifestyle associated diseases, there were conflicting responses when enquired about the genetic predisposition to obesity. This showed that most of the participants were aware that obesity could be prevented but were very unsure whether it could be prevented if someone had a family history of obesity. This is an area were further community education and school health programs could focus to remove this false perception. This could lead to further reduction in prevalence of obesity and a healthier community.

\section{References}

[1]. WHO; obesity and overweight. www.who.int/mediacentre/factsheets/fs311/en (accessed on 11th January 2015)

[2]. Reddy MN, Kumar CK, Jamil K. New World Syndrome (Obesity) in South India. Cited 2012 Dec. http://omicsonline.org/scientificreports/2165-7904-SR-567.pdf

[3]. Bray GA. Complications of obesity. Annals of internal medicine. 1985 Dec 1;103(6_Part_2):1052-62.

[4]. Ogden CL, Carroll MD, Flegal KM. Epidemiologic trends in overweight and obesity. Endocrinology and metabolism clinics. 2003 Dec 1; 32(4):741-60.

[5]. Klein S, Fabbrini E, Romijn JA. Obesity. In: Melmed S, Polonsky KS, Larsen PR, Kronenberg HM. William's Textbook of Endocrinology, $12^{\text {th }}$ ed. Philadelphia: Elsevier/Saunders;2012.p.1605-32. 